\title{
Serum creatine kinase and lactic dehydrogenase levels as useful markers of immediate and long-term outcome of perinatal asphyxia
}

\author{
D H Karunatilaka ${ }^{1}$, G W D S Amaratunga ${ }^{2}$, K D N I Perera ${ }^{3}$, V Caldera ${ }^{4}$
}

Sri Lanka Journal of Child Health, 2000; 29: 49-52

(Key words: creatine kinase, lactic dehydrogenase, markers of perinatal asphyxia)

\begin{abstract}
Objectives To determine the usefulness of creatine kinase (CK) alone or in combination with lactic dehydrogenase (LDH) in identifying, those infants at risk of developing hypoxic ischaemic encephalopathy (HIE) or a major handicap following perinatal asphyxia.
\end{abstract}

Design Prospective observational study.

Method CK and LDH in serum were measured in 35 asphyxiated infants and in 30 controls within 6 hours of delivery. The asphyxiated newborns were monitored for evidence of HIE. Both groups were followed up in paediatric outpatients clinic and a developmental assessment was done at the end of one year.

Results Both the $\mathrm{CK}$ and $\mathrm{LDH}$ levels were significantly raised in all asphyxiated newborns. $25.7 \%$ of asphyxiated babies developed HIE. The CK and LDH values of those who developed HIE were significantly higher than the rest of the asphyxiated newborns. Five babies had developmental delay and their $\mathrm{CK}$ values too were significantly higher. The same relationship was not seen with their $\mathrm{LDH}$ values.

Conclusion Both the $\mathrm{CK}$ and $\mathrm{LDH}$ values are raised in birth asphyxia. The increase is more marked among those who developed HIE. However, only the raised CK levels correlated with long-term outcome. Babies with CK values above 2860 IU/ L should be closely monitored for both the immediate and longterm sequelae.

\section{Introduction}

Prediction of outcome of perinatal asphyxia is important but formidable ${ }^{1}$. The Apgar score has a limited role in predicting the immediate outcome,

${ }^{1}$ Consultant Paediatrician, ${ }^{2}$ Registrar, ${ }^{3}$ Senior House Officer, Paediatric Unit, Colombo South Teaching Hospital. such as that of Hypoxic Ischaemic Encephalopathy (HIE) and the long-term sequelae ${ }^{2}$. Several studies have shown that cerebral function monitoring (CFM) using non invasive techniques, such as EEG within six hours of birth, cranial ultrasonography, cranial topography, Doppler measurements of cerebral blood flow, somato-sensory evoked potentials, magnetic resonance imaging and estimation of neurophysiological markers such as brain specific creatine kinase (CK-BB), brain specific lactic dehydrogenase isomer, glutamate and neurone specific enolase in the CSF are all useful in predicting both the immediate dysfunction and the long term outcome ${ }^{1,3,4}$. But none of these facilities are routinely available, even in the teaching hospitals of this country.

A "therapeutic window" exists in the early hours following asphyxia and perhaps for a longer period, when intervention can attenuate activation of the neurotoxic cascade that leads to ultimate cell death, either hours or days later ${ }^{6}$. Although the precise mechanisms of neuronal loss are unclear, both apoptosis and necrosis seem to contribute to causing cell death following hypoxic ischaemia.

Several neuro-protective strategies to improve cerebral outcome after birth asphyxia have been published and there are a few ongoing multicentre trials, which may prove useful in neuronal rescue, if used early, within the crucial therapeutic window period $^{7,8,9}$. All such therapeutic modalities must be undertaken with great caution in view of the possible side effects. Therefore, only the infants at risk of developing HIE, and potential brain damage should be considered for such treatment.

\section{Objectives}

Since CFM and the measurements of neurophysiological markers mentioned above are not routinely available in Sri Lanka, we examined the serum levels of $\mathrm{CK}$ and $\mathrm{LDH}$ to determine whether $\mathrm{CK}$ level alone or in combination with LDH level could be used as an indicator in selecting at a very early stage, those infants at risk of developing HIE or long term sequelae such as neurological abnormality 
or developmental delay. Several workers have previously demonstrated that neuron specific isomers of both these enzymes are released in large quantities into the circulation following anoxic injury from the many tissues of their storage and are reflected in total serum levels ${ }^{10,11}$. However, no attempt was made to show any causal relationship to the outcome of asphyxia as they were better equipped to measure the brain specific isomers. The sites of storage of CK are the brain (CKBB), skeletal muscle (CKMM) and the heart muscle (CKMB) and the sites of storage of IDH are the brain, the kidney, the heart, skeletal muscle and the erythrocytes.

\section{Method}

This prospective study included 35 term infants $(>37$ weeks of gestational age) born at Teaching Hospital Colombo South between June 97 - November 97 with perinatal asphyxia and having two or more of the following criteria; 1) Apgar score of $<5$ at 5 minutes, 2) failure to establish spontaneous respiration, 3) requirement of IPPV for at least 3 minutes, 4) fetal heart rate $<100$ beats per minute, 5) meconium stained liquor, 6) deceleration of fetal heart shown on fetal heart monitor, 7) umbilical arterial $\mathrm{pH}<7$ or base deficit $>10 \mathrm{mmol} / \mathrm{L}^{1,5}$. A control group consisted of 30 non-asphyxiated newborns, each of whom was born around the same time as a baby in the study group. Permission to bleed normal nonasphyxiated infants was obtained after adequately explaining the purpose and the procedure to the respective mothers and their consent was recorded on case notes. The Director of the Colombo South Teaching Hospital gave permission for the study and the consent of the two obstetricians was obtained.

All 65 babies (in both groups) were examined by one of the four investigators prior to obtaining blood samples. Blood samples for $\mathrm{CK}$ and $\mathrm{LDH}$ were collected within the first six hours after birth and all samples were immediately despatched to the analysing laboratory by prior arrangement. The usual measures were taken to avoid haemolysis of the samples collected. Twenty-six of the thirty five asphyxiated newborns were admitted to the special care baby unit for initial assessment and observation, while nine babies who appeared well after initial resuscitation remained with their mothers in the postnatal ward. All 35 were closely monitored during the next three days for any evidence of HIE. Those who showed evidence of HIE were further categorised clinically, into mild moderate and severe based on Sarnet and Sarnet classification ${ }^{12}$.

\section{Clinical classification of $\mathrm{HIE}^{12}$}

\begin{tabular}{|l|l|}
\hline Mild & $\begin{array}{l}\text { Hyperalertness, minor } \\
\text { disturbances of tone, some } \\
\text { feeding difficulty and recovery } \\
\text { in } 48 \text { hours. }\end{array}$ \\
\hline Moderate & $\begin{array}{l}\text { Lethargy, more pronounced } \\
\text { abnormalities of tone, poor } \\
\text { feeding and convulsions with } \\
\text { recovery within 7 days }\end{array}$ \\
\hline Severe & $\begin{array}{l}\text { Coma, failure to maintain } \\
\text { adequate ventilation, profound } \\
\text { hypotonia and seizures. }\end{array}$ \\
\hline
\end{tabular}

Babies in the study group and in the control group were followed up in paediatric outpatients clinic for a period of one year and a detailed neurological and a developmental assessment based on Denver developmental criteria were done at the end of this period. The values of CK and LDH in both groups were later compared with the immediate and the long-term outcome at one year.

\section{Results}

Thirty-one infants in the asphyxiated group (88.5\%) and twenty-four infants in the control group (80\%) completed the study. Four in the asphyxiated (11.4\%) and six $(20 \%)$ in the control group were lost to follow up.

Table 1 shows the comparison of serum CK and LDH values among the asphyxiated and healthy babies. The median values of both the $\mathrm{CK}$ and $\mathrm{LDH}$ are significantly higher in asphyxiated newborns. Table 2 shows the immediate outcome of asphyxiated babies. 9 out of $35(25.7 \%)$ babies in the asphyxiated group developed HIE based on Sarnet and Sarnet criteria. Table 3 shows that the CK and LDH values of those who developed HIE are significantly higher than the rest of the asphyxiated group who did not develop HIE. 
Table 1

Median CK and LDH values (IU/L) among asphyxiated and non-asphyxiated babies

\begin{tabular}{|l|l|l|}
\hline \multicolumn{1}{|c|}{ Category of babies } & \multicolumn{1}{|c|}{$C K^{1}$} & \multicolumn{1}{c|}{$L^{1}$} \\
\hline Asphyxiated & 1824.0 & 2948.0 \\
$(\mathrm{n}=35)$ & $(807.8-2976.0)$ & $(1505.0-4256.0)$ \\
\hline Non asphyxiated & 443.3 & 1671.2 \\
$(\mathrm{n}=30)$ & $(388.6-512.0)$ & $(1326.0-2406.2)$ \\
\hline
\end{tabular}

All values given are the median (25th -75 th centiles)

${ }^{1}$ Kruskal- Wallis $=31.87, \mathrm{df}=1, \mathrm{P}=0.0000001$

${ }^{2}$ Kruskal- Wallis $=5.99, \mathrm{df}=1, \mathrm{P}=0.01$

Table 2

\begin{tabular}{|l|c|c|}
\hline \multicolumn{2}{|c|}{ Table 2 } \\
\hline \multicolumn{1}{|c|}{ Immediate outcome of asphyxiated babies } \\
\hline \multicolumn{1}{|c|}{ Outcome } & Number & $\%$ \\
\hline Remained normal after asphyxia & 26 & 74.3 \\
\hline Developed HIE & 9 & 25.7 \\
\hline
\end{tabular}

\section{Table 3}

\begin{tabular}{|l|c|c|}
\hline \multicolumn{2}{|c|}{ Median CK and LDH values (IU/L) in relation to outcome of asphyxiated babies } \\
\hline \multicolumn{1}{|c|}{ Outcome } & $C K^{l}$ & $L^{2}$ \\
\hline Remained normal & 953.6 & 2395.3 \\
\hline after asphyxia $(\mathrm{n}=26)$ & $(725.2-1995.1)$ & $(1279.7-3962.0)$ \\
\hline Developed HIE & 5988.8 & 4246.0 \\
\hline$(\mathrm{n}=9)$ & $(4132.0-6420.00)$ & $(2742.2-5780.6)$ \\
\hline
\end{tabular}

All values given are the median (25th - 75th centiles)

${ }^{1}$ Kruskal- Wallis $=19.5, \mathrm{df}=1, \mathrm{P}=0.00001$

${ }^{2}$ Kruskal- Wallis $=5.13, \mathrm{df}=1, \mathrm{P}=0.02$

Out of the nine babies who developed HIE (Table 4), the one who developed severe HIE succumbed on the 5th day. Three had moderately severe HIE while five had mild HIE. An upward trend in both the $\mathrm{CK}$ and $\mathrm{LDH}$ values with the increasing severity of HIE was seen but this was not statistically significant.

\begin{tabular}{|l|c|c|}
\hline \multicolumn{3}{|c|}{ Table 4 } \\
\hline \multicolumn{3}{|c|}{ Severity of HIE in relation to median values } \\
\hline \multicolumn{2}{|c|}{ CK and LDH values (IU/L) } \\
\hline Severity of HIE & $C K^{l}$ & $L D H^{2}$ \\
\hline Mild $(\mathrm{n}=5)$ & 4132.0 & 3876.0 \\
\hline & $(3200.0-4260.3)$ & $(2742.2-4246.0)$ \\
\hline Moderate $(\mathrm{n}=3)$ & 6066.6 & 5505.0 \\
\hline & $(5988.8-6420.0)$ & $(1290.0-5860.0)$ \\
\hline Severe $(\mathrm{n}=1)$ & 8222.0 & 7653.0 \\
\hline
\end{tabular}

All values given are the median ( 25 th -75 th centiles)

${ }^{1}$ Kruskal-Wallis $=3.84, \mathrm{df}=2, \mathrm{P}=0.15$

${ }^{2}$ Kruskal- Wallis $=2.56, \mathrm{df}=2, \mathrm{P}=0.28$ 
Of the 31 asphyxiated infants finally assessed at one year, five showed a delay in more than one sphere of development. These included all three babies who had moderate HIE and one baby who had mild HIE. The other baby who showed developmental delay did not have HIE but was a product of a consanguineous marriage. One who developed moderate HIE also had mild microcephaly and had cerebral palsy. Another who developed mild HIE had a convergent squint. These two babies were detected during follow up at three months of age and were already receiving treatment. All infants in the control group showed normal neurodevelopment, when assessed at one year.

\begin{tabular}{|l|c|c|}
\hline \multicolumn{3}{|c|}{ Table 5 } \\
\hline \multicolumn{3}{|c|}{ Long term sequel of asphyxia in relation to } \\
\hline \multicolumn{3}{|c|}{ median CK and LDH values (IU/L) } \\
\hline Severity of HIE & $C K^{l}$ & $L D H^{2}$ \\
\hline Delayed development & 5988.8 & 3156.4 \\
\hline following HIE (n=5) & $(4132.0-6066.6)$ & $(2742.3-5505.0)$ \\
\hline Normal development & 3200.0 & 3876.0 \\
\hline despite mod. HIE (n=3) & $(2976.0-6896.0)$ & $(2428.0-4246.0)$ \\
\hline Normal development & 956.6 & 2440.2 \\
\hline without HIE $(\mathrm{n}=23)$ & $(752.2-1995.1)$ & $(1505.0-4226.0)$ \\
\hline
\end{tabular}

All values given are the median (25th -75 th centiles $)$

${ }^{1}$ Kruskal- Wallis $=15.79, \mathrm{df}=2, \mathrm{P}=0.0004$

${ }^{2}$ Kruskal- Wallis $=1.72, \mathrm{df}=2, \mathrm{P}=0.42$

Table 5 illustrates the long-term outcome of birth asphyxia in relation to $\mathrm{CK}$ and $\mathrm{LDH}$ values. The $\mathrm{CK}$ values appear to be highest among those who developed HIE and ended up having developmental delay. The CK values of those who had normal development despite initial HIE were also significantly higher when compared to those who had normal development despite initial asphyxia. This observation is highly significant but the same trend is not seen with the LDH values.

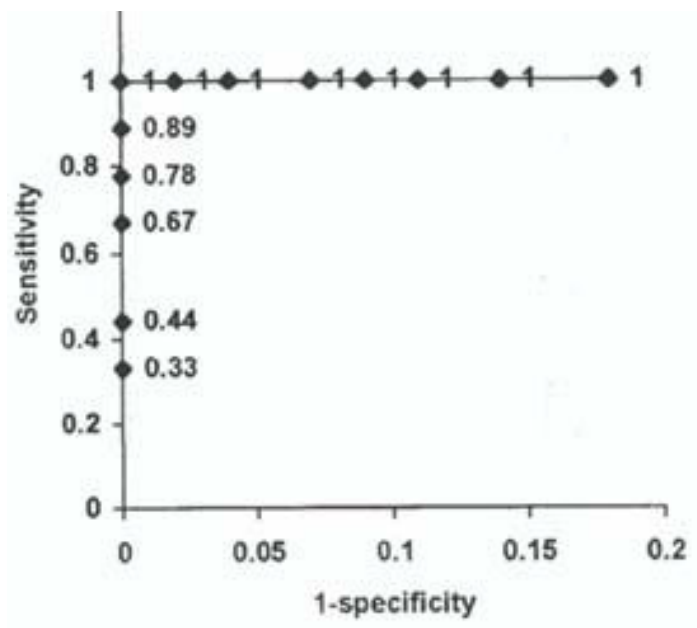

Figure 1. ROC curve with Creatine kinase values
As the observations with regard to $\mathrm{CK}$ values (Tables $1,3,5)$ were significant in detecting both the immediate and the long-term outcome of asphyxia, a ROC curve (Figure 1) was drawn in order to estimate the cut off value for $\mathrm{CK}$ in significant birth asphyxia. According to the ROC curve the most appropriate cut off point for CK appears to be at $2860 \mathrm{IU} / \mathrm{L}$.

\section{Discussion}

Birth asphyxia is still a major problem in this country and it undoubtedly contributes towards significant morbidity in the immediate neonatal period and in late infancy. We have shown that the serum CK level in particular and also LDH level in the first six hours, though not the best predictors of outcome offers us sufficient indication about those babies who will be developing HIE between 6-72 hours after birth. Thus active therapeutic intervention may be offered to them before the cascade of neurochemicals unleash their effects. However, only the CK values showed a causal relationship with the long term outcome and this relationship was not evident with the $\mathrm{LDH}$ values.

\section{Conclusions}

We conclude that both the CK and LDH values are raised in birth asphyxia and the increase is more 
marked among those who developed HIE as a result. The long-term sequelae of birth asphyxia showed a positive relationship only with the raised levels of $\mathrm{CK}$ and not with the LDH levels. Therefore the measurement of serum CK of asphyxiated newborns is useful as a screening test in our country and the babies with serum levels of CK above $2860 \mathrm{IU} / \mathrm{L}$ within the first six hours of birth should be closely monitored for the possible development of HIE and should also be given a guarded prognosis.

\section{Acknowledgements}

We acknowledge the assistance given with the statistical analysis by Dr Renuka Jayatissa, consultant nutritionist of the Medical Research Institute and the house officers and the nursing staff of the special care baby unit for their enthusiastic participation. MeadJohnson Nutritionals funded this study.

\section{References}

1. Thornberg E, Thiringer $\mathrm{K}$, Hagberg $\mathrm{H}$, et al. Neurone specific enolase in asphyxiated newborns: Association with encephalopathy and cerebral function monitor trace. Archives of Disease in Childhood 1995; 72: 39-42.

2. Mlevene M 1, Caroline S, Grindulis H, Moore J R. Comparision of two methods of predicting outcome in perinatal asphyxia. Lancet 1986; 1: 6770 .

3. Ekin P, Toet M C, Groeneedaal F, et al. Predictive value of neuroimaging, pulse Dopplerand neurophysiology in full term infants with hypoxic ischaemic encephalopathy. Archives of Disease in Childhood 1995; 73: 75-80

4. Bjerre 1, Hellstrom- Westas, Rosen 1, et al. Monitoring of cerebral function after severe asphyxia in infancy. Archives of Disease in Childhood 1983; 58: 997-1002.
5. Levine M, Blennow M, Whitelaw A, Feliman V. Acute effects of two different doses of magnesium sulphate in infants with birth asphyxia. Archives of Disease in Childhood 1995; 73:174-7.

6. Gluckman P D, Williams C E. When and why do brain cells die? Dev. Med. Child Neurology 1992; 34:1010-4.

7. Vnnucci R C, Perlman J M. Interventions for perinatal ischaemic encephalopathy. Paediatrics 1997; 100: 1004-14.

8. Tan S, Parks D A. Preserving brain function during neonatal asphyxia. Clin. Perinatology 1999; 26: 733-47.

9. Robertson N J, Edwards AD. Recent advances in developing neuroprotective strategies for perinatal asphyxia. Current Opin. Pediatrics 1998; 10: 57580 .

10. De Praeter C, Vanhaesebrouck P, Govaert P, et al. Creatinine Kinase Isoenzyme BB concentrations in the cerebrospinal fluid of newborns. Pediatrics 1991; 88:1204-10.

11. Fernandez F, Verdu A, Quero J. A serum CPK-BB Isoenzyme in the assessment of brain damage in asphyctic term infants. Acta Paeditrica. Scand. 1987; 76: 914-8.

12. Sarnet H B, Sarnet M S. Neonatal encephalopathy following fetal distress. Arch. Neurology 1976, 696-705. 